\title{
Demo Abstract: Towards IoT Service Deployments on Edge Community Network Microclouds
}

\author{
Amin M. Khan*§, Felix Freitag ${ }^{\dagger}$, Vladimir Vlassov ${ }^{\ddagger}$, Phuong Hoai Ha* \\ * Department of Computer Science, UiT The Arctic University of Norway. Troms $\varnothing$, Norway \\ \{amin.khan, phuong.hoai.ha\}@uit.no \\ $\dagger$ Department of Computer Architecture, Universitat Politècnica de Catalunya. Barcelona, Spain \\ felix@ac.upc.edu \\ $\ddagger$ KTH Royal Institute of Technology. Stockholm, Sweden. \\ vladv@kth.se \\ $\S$ Hitachi Vantara Corporation. Lisbon, Portugal
}

\begin{abstract}
Internet of Things (IoT) services for personal devices and smart homes provided by commercial solutions are typically proprietary and closed. These services provide little control to the end users, for instance to take ownership of their data and enabling services, which hinders these solutions' wider acceptance. In this demo paper, we argue for an approach to deploy professional IoT services on user-controlled infrastructure at the network edge. The users would benefit from the ability to choose the most suitable service from different IoT service offerings, like the one which satisfies their privacy requirements, and third-party service providers could offer more tailored IoT services at customer premises. We conduct the demonstration on microclouds, which have been built with the Cloudy platform in the Guifi.net community network. The demonstration is conducted from the perspective of end users, who wish to deploy professional IoT data management and analytics services in volunteer microclouds.
\end{abstract}

Index Terms-edge cloud computing; community clouds

\section{INTRODUCTION}

In this demo paper, we aim to show the concept of an open edge infrastructure for professional Internet of Things (IoT) service deployment by demonstrating that in a user-controlled system, enabled by the Cloudy platform ${ }^{1}$, IoT services can be successfully run in volunteer-driven microclouds. Important use cases of the IoT with huge business potential have driven edge computing research and deployment [1]. Edge computing solutions are nowadays used in many industrial and consumeroriented scenarios, covering major application domains [2]. Many of the solutions currently on the market, however, cannot be easily extended by framework-independent thirdparty services.

Many concepts and implementations, on which we build our edge computing approach presented in this demonstration, are currently developed or have been successfully proven in the past. Container-based service provisioning on Customer Premises Equipment (CPE) is proposed in the ParaDrop system [3], and container toolkits and standardization are nowadays heavily promoted by the Linux Foundation's project,

${ }^{1}$ https://cloudy.community/

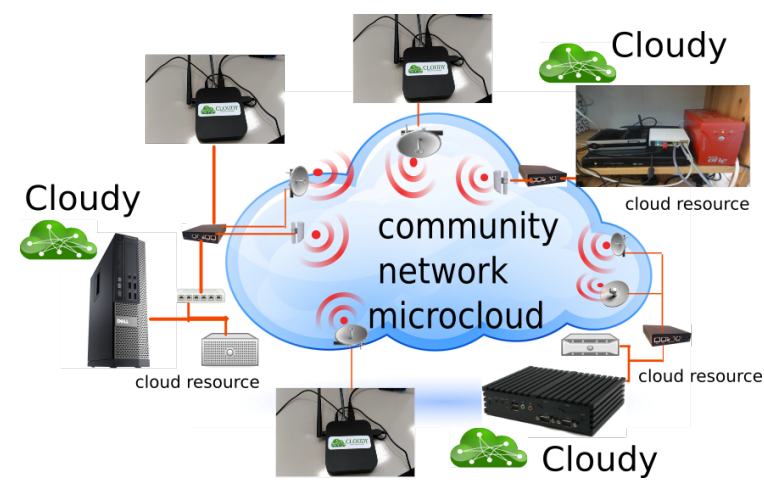

Figure 1. Mircocloud with heterogeneous infrastructure.

Cloud Native Computing Foundation (CNCF). Data processing for IoT, through conducting data-intensive computing on distributed and decentralized clouds at the network edge [4], has been under active research. The contribution to volunteer computing was demonstrated by applications such as Folding@home [5], where a large number of participants donated computing resources to support research.

An open platform to deploy IoT services lets us envision a market for more customized edge services, which would not only benefit the end users, but also lead to new business opportunities for service providers, who can avoid providing their own IoT gateways, and instead run their services directly on users' commodity devices.

\section{IOT SERVICES IN MICROCLOUDS}

We demonstrate our approach by building the use case of a professional IoT service, which is available both as a commercial enterprise edition and an open-source community edition. The environment we use for the demonstration is a microcloud deployed in the Guifi.net community network ${ }^{2}$. These microclouds consist of distributed heterogeneous computing devices contributed by the network participants [6], [7]. Such devices are typically inexpensive mini-PCs or virtual machines

${ }^{2}$ https://guifi.net/

(C) 2018 IEEE. Personal use of this material is permitted. Permission from IEEE must be obtained for all other uses, in any current or future media, including reprinting/republishing this material for advertising or promotional purposes, creating new collective works, for resale or redistribution to servers or lists, or reuse of any copyrighted component of this work in other works. DOI 10.1109/INFCOMW.2018.8406840 


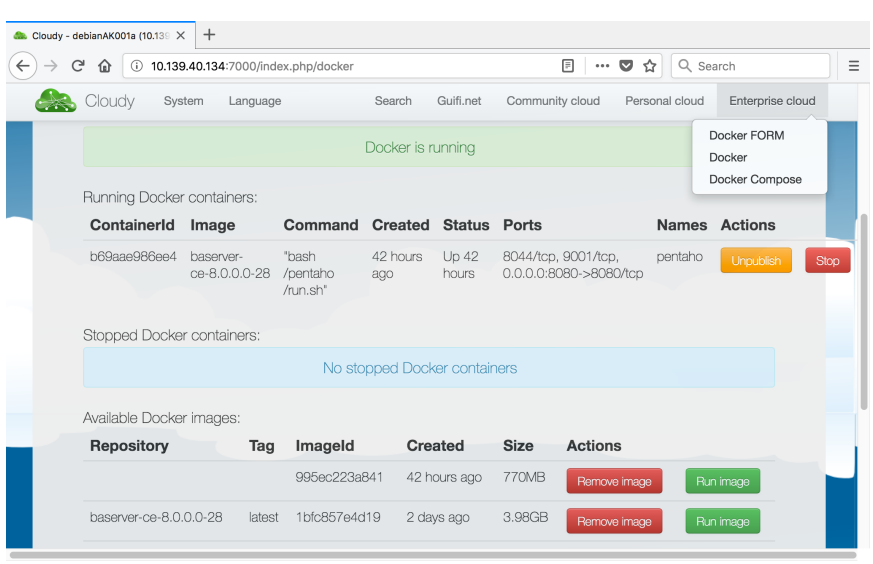

Figure 2. Pentaho IoT data analytics services in Cloudy.

(VMs), which run the Cloudy software. Figure 1 illustrates these community network microclouds.

This open deployment environment is represented by the Cloudy distribution, which has been developed to build community microclouds. Cloudy is installed by the users on their edge devices. Through the support services of Cloudy, a new edge node is integrated into the existing community microcloud. Cloudy foresees the deployment of third-party applications as Docker containers.

To represent the professional IoT services, we have chosen Hitachi Vantara's Pentaho open-source data analytics and business intelligence (BI) platform [8]. Pentaho provides comprehensive data processing, integration, reporting and mining features. Users can design data workflows, reports and dashboards, which are uploaded to Pentaho Server for processing. Pentaho Server also integrates support for Weka, a popular open-source data mining software, as well as Python and R for executing machine learning programs.

We deploy the Pentaho Server as a Docker container on Cloudy nodes. In this scenario, owners of the edge devices set up Pentaho Server instances, and provide these IoT data management and analytics services to the other users of the community microcloud. For data collection and storage, the users can have a virtual machine with a public cloud provider on the Internet, or set up another node in the community microcloud as an FTP or a database server for storing the data received from IoT devices. For instance in our setup, we deploy the PostgreSQL database running as a Docker container on a Cloudy device.

Figure 2 shows through the Cloudy's web-based management interface the Pentaho Server running as a Docker container after the installation of the service in Cloudy. The service is discoverable in the microcloud since the provider has tagged to publish it. Therefore any participant of the community cloud will be able to find it, and can access the Pentaho Server at IP address 10.139.40.134.

After logging in by accessing the service through the Cloudy web interface, the user sees the home screen of the Pentaho Server. With the credentials given by the service provider, the

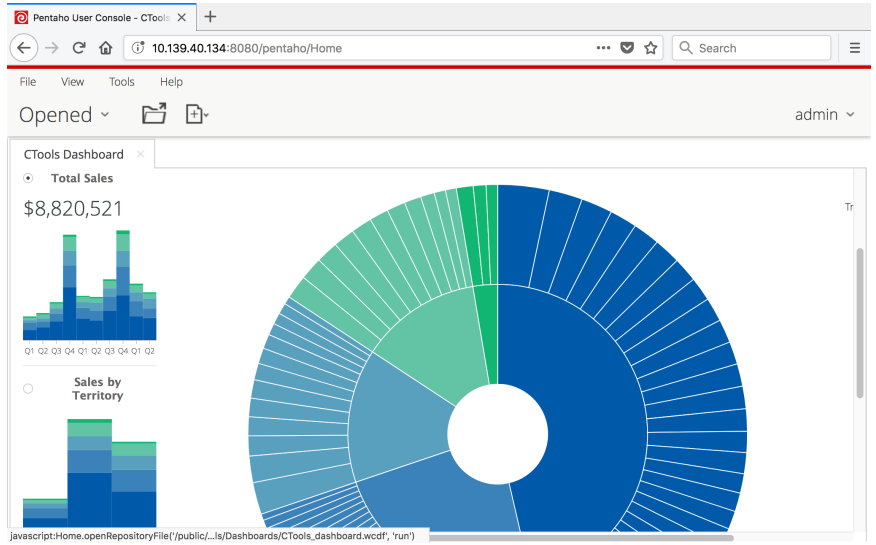

Figure 3. Pentaho server in microcloud with data dashboards.

user can access and manage stored files, schedule jobs, and generate data reports and dashboard as shown in Figure 3.

\section{EXPERIMENTATION}

The experimentation in this demo aims to show the capability of installing professional IoT services in an open edge computing platform represented by Cloudy, and consists of:

1) Exploration of the microcloud by live access to a Cloudy node ${ }^{3}$.

2) Through the web interface of Cloudy, managing and accessing specific services from the perspective of an end user.

3) Exploring the support services of Cloudy which enable service publication and discovery, node management and update.

\section{ACKNOWLEDGMENT}

This work is supported by the European Union's Horizon 2020 programme under netCommons project (H2020-688768), by the Research Council of Norway under PREAPP project (231746/F20), by the Spanish government under contract TIN2016-77836-C2-2-R, and by the KTH Royal Institute of Technology, Stockholm, Sweden.

\section{REFERENCES}

[1] W. Shi and S. Dustdar, "The Promise of Edge Computing," Computer, vol. 49, no. 5, pp. 78-81, 2016.

[2] "Azure and the Internet of Things." [Online]. Available: https://docs.microsoft.com/en-us/azure/iot-suite/

[3] P. Liu, L. Hartung, and S. Banerjee, "Lightweight Multitenancy at the Network's Extreme Edge," Computer, vol. 50, no. 10, pp. 50-57, 2017.

[4] A. Chandra, J. Weissman, and B. Heintz, "Decentralized Edge Clouds," IEEE Internet Computing, vol. 17, no. 5, pp. 70-73, 2013.

[5] A. L. Beberg et al., "Folding@home: Lessons From Eight Years of Volunteer Distributed Computing," in HiCOMB '09, within IPDPS, 2009

[6] M. Selimi et al., "Practical Service Placement Approach for Microservices Architecture," in CCGrid, May 2017, pp. 401-410.

[7] A. M. Khan and F. Freitag, "On Edge Cloud Service Provision with Distributed Home Servers," in CloudCom, Dec. 2017, pp. 223-226.

[8] M. R. Casters, R. Bouman, and J. van. Dongen, Pentaho Kettle Solutions: Building Open Source ETL Solutions with Pentaho Data Integration. Wiley, 2010.

\footnotetext{
${ }^{3}$ Access is available through a publicly available Cloudy instance at http://demo.cloudy.clommunity/, with login guest:guest.
} 